\title{
An Articulated Statistical Shape Model of the Human Knee
}

\author{
Matthias Bindernagel, Dagmar Kainmueller, Heiko Seim, Hans Lamecker, \\ Stefan Zachow, Hans-Christian Hege \\ Zuse Institute Berlin \\ bindernagel@zib.de
}

\begin{abstract}
In this work we present an articulated statistical shape model (ASSM) of the human knee. The model incorporates statistical shape variation plus explicit degrees of freedom that model physiological joint motion. We also present a strategy for segmentation of the knee joint from medical image data. We show the potential of the model via an evaluation on a set of 40 clinical MRI datasets with manual expert segmentations available.
\end{abstract}

\section{Introduction}

For biomechanical analysis or surgery planning it can be beneficial to reconstruct an estimated healthy joint anatomy from medical image data in the presence of strong pathological changes or implants. For total knee arthroplasty, for instance, knowledge about the morphology of the joint before osteoarthritis may impact the choice of implant. Since the respective image data is typically not available, the need for an estimation from the pathological case arises. Secondly, an accurate reconstruction of the actual patient-specific joint anatomy is often needed.

Statistical shape models (SSMs) [1] are a powerful tool for reconstructing both estimated healthy as well as actual patient-specific anatomies. Multiple SSMs of different bones may be applied successfully for the reconstruction of joints [2]. However, such an approach does not model knowledge about joint posture or correlated morphology of the involved bones, and consequently lacks robustness. Robustness is crucial for the reconstruction of the estimated healthy anatomy, as the pathological or implanted region in the image data, which usually provides significant information about the joint morphology, is deliberately ignored here. In case of poor contrast in the joint region, robustness also plays an important role for the reconstruction of actual patient-specific anatomies.

One may model joint flexibility implicitly by capturing joint motion statistically $[3,4]$. This approach is beneficial only if relative transformations between individual objects are a statistical property of anatomy, which is, e.g., not the case for knee bending. Instead, we follow the approach presented for the hip joint in [5] and propose an articulated SSM (ASSM) of the knee, where we 
model knee joint posture explicitly as a combination of characteristic transformations [6]. With an evaluation on 40 MRI datasets, we show that our knee ASSM outperforms reconstruction based on separate SSMs.

\section{Materials and Methods}

\subsection{Model of the Knee Joint}

Our knee joint model consists of the femur (thighbone) and the tibia (shinbone), represented by triangular surface meshes. As proposed in [6] we model the joint motion by (i) a rotation around the epicondylar axis, which is defined by the lateral and medial epicondyles of the femur that are represented as vertices of the femur mesh, and (ii) a translation in direction of the intersection of the tibial plateau and the epicondylar axis shape model of the human knee normal plane (Fig. 1a). Accordingly, the transformation $K$ of the tibia relative to the femur can be written as

$$
K(\alpha, t)=\operatorname{rot}\left(\boldsymbol{r}_{\text {epi }}, \boldsymbol{d}_{\text {epi }}, \alpha\right) \circ \operatorname{trans}\left(\boldsymbol{d}_{\text {plateau }}, t\right)
$$

where rot denotes the rotation around the epicondylar axis with origin $\boldsymbol{r}_{\text {epi }}$ and direction $\boldsymbol{d}_{\text {epi }}$ by an angle $\alpha$, and trans represents a translation in direction of $\boldsymbol{d}_{\text {plateau }}$ by a distance $t$.

In addition to the global transformation $T_{g}$ of the knee bone compound the model incorporates a local transformation $K$ for the tibia to keep track of varying joint postures. That is, the overall transformation of the femur is $T_{g}$ and the overall transformation of the tibia equals $T_{g} \circ K . K=I$ represents a reference bending. To adjust the knee posture to a particular $\alpha$ and $t$ under motion of the tibia, one has to set $K \leftarrow K(\alpha, t)$. In order to adjust the joint under motion of the femur, one may apply $T_{g} \leftarrow T_{g} \circ K^{-1}(\alpha, t)$ and $K \leftarrow K(\alpha, t)$.

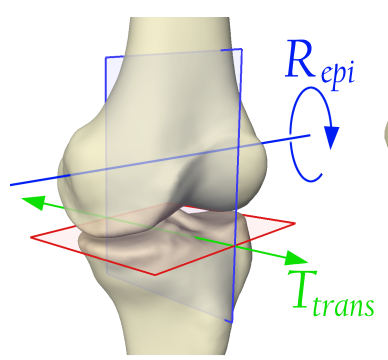

(a)

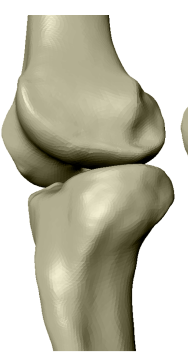

(b)

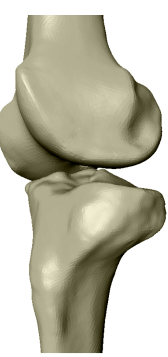

(c)

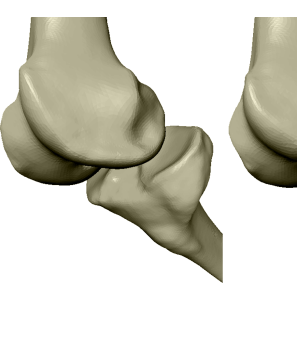

(d)

Fig. 1. Knee joint motion is modeled by rotation $R_{\text {epi }}$ around the epicondylar axis and translation $T_{\text {trans }}(\mathrm{a})$. Instances of the knee ASSM: Shape and joint state I (b), shape II and joint state I (c), shape I and joint state II (d), and shape and joint state II (e). 


\subsection{Adjusting Transformations by Alignment of Bones}

We employ the root mean square distance of all vertices $\boldsymbol{v}_{i}$ of a given model instance to corresponding vertices $\boldsymbol{v}_{\text {ref }, i}$ of a reference mesh to measure alignment

$$
D\left(T_{g}, K\right)=\sum_{i \in I_{\mathrm{femur}}}\left\|T_{g} \circ \boldsymbol{v}_{i}-\boldsymbol{v}_{\mathrm{ref}, i}\right\|^{2}+\sum_{i \in I_{\mathrm{tibia}}}\left\|T_{g} \circ K \circ \boldsymbol{v}_{i}-\boldsymbol{v}_{\mathrm{ref}, i}\right\|^{2}
$$

where $I_{\text {femur }}$ and $I_{\text {tibia }}$ are the sets of vertex indices of the femur and tibia, respectively. Hence, to align a model instance to a reference mesh in terms of transformation, one must optimize $D\left(T_{g}, K\right)$ with respect to $T_{g}$ and $K$.

We propose an iterative scheme to optimize $T_{g}$ and $K$ by repeated alignment of the object compound, the femur, and the tibia until $\Delta D$ falls below a userdefined stopping criterion. Here, one iteration is composed of a sequence of alignment steps: One may, e.g., align the whole compound, then align the tibia by joint adjustment while keeping the femur fixed, and then align the femur by joint adjustment while keeping the tibia fixed (sequence CTF), align the whole compound with regard to femur vertices only, and then align the tibia by joint adjustment (sequence $\mathrm{C}_{\mathrm{F}} \mathrm{T}$ ), or vice versa (sequence $\mathrm{C}_{\mathrm{T}} \mathrm{F}$ ).

The object compound is aligned via rigid and scale transformations. For a description of well-established methods [1]. To align femur and tibia by means of knee joint adjustment, one has to find

$$
\begin{gathered}
\underset{\alpha, t}{\operatorname{argmin}}\left[D_{\text {femur }}(\alpha, t)=\sum_{i \in I_{\text {femur }}}\left\|T_{g} \circ K^{-1}(\alpha, t) \circ \boldsymbol{v}_{i}-\boldsymbol{v}_{\text {ref }, i}\right\|^{2}\right] \\
\underset{\alpha, t}{\operatorname{argmin}}\left[D_{\text {tibia }}(\alpha, t)=\sum_{i \in I_{\text {tibia }}}\left\|T_{g} \circ K(\alpha, t) \circ \boldsymbol{v}_{i}-\boldsymbol{v}_{\text {ref }, i}\right\|^{2}\right]
\end{gathered}
$$

for femur and tibia, respectively. A simple and efficient way to solve these problems is by iterative optimization with respect to $\alpha$ and $t$ separately, since the respective partial derivatives of $D_{\text {femur }}(\alpha, t)$ and $D_{\text {tibia }}(\alpha, t)$ as well as their roots can be computed analytically. Optimization is stopped when $\Delta D$ falls below a user-defined convergence criterion.

\subsection{ASSM Generation}

We generated the articulated knee model from 40 training shapes made available by [7]. In contrast to the generation of SSMs of single objects, the preparation of training shapes for an ASSM requires alignment via transformations according to the joint model. To this end, we use the method described in Sec. 2.2, after we have determined vertex correspondences on the set of training shapes as in [2]. We then generate the ASSM by applying principal component analysis on the aligned and corresponding training meshes. An example of varying joint posture and shape is shown in Fig. 1, b-e. 
Fig. 2. Results. Contour: separate SSMs (black) and ASSM (white); rectangle: extrapolated purely from the model (white).
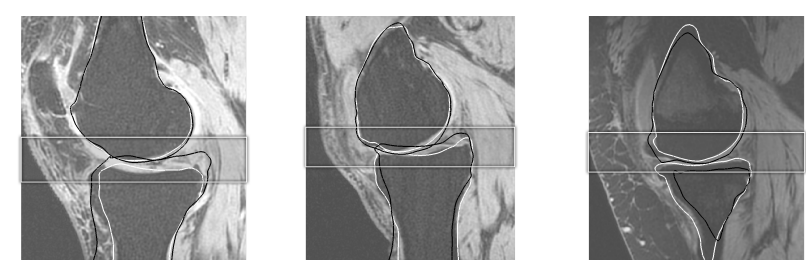

\subsection{Segmentation Framework}

Commonly, an iterative segmentation process repeats the following steps to adjust an SSM to image data:

1. Analyze the normal intensity profiles of the current SSM instance within the image data. Here, we employ the strategy presented in [2].

2. Displace vertices of the instance to positions that better fit the image data, resulting in a triangular mesh that is in general not an instance of the SSM.

3. Adjust the overall transformation of the instance to fit the displaced mesh.

4. Adjust shape parameters of the instance to fit the displaced mesh.

In order to cope with the relative joint transformation we extend step 3 to adjust not only the overall transformation of the compound shape, but also the relative transformation within the joint, as proposed in Sec. 2.2. Note that in general the shape adjustment in step 4 changes the transformation axes of the model, which results in a slight change of the relative transformation. This effect is currently only compensated for by the iterative nature of the approach.

\section{Results}

To evaluate the model with regard to different alignment sequences as suggested in Sec. 2.2, we initially built three different ASSMs: For each ASSM, the training shapes were aligned according to one of the three sequences CTF, $\mathrm{C}_{\mathrm{F}} \mathrm{T}$ and $\mathrm{C}_{\mathrm{F}} \mathrm{T}$. We conducted a leave-one-out study for each model, using the respective "leftout" training shape in its unaligned form as target. There are no significant differences among the three ASSMs in terms of average reconstruction accuracy. However, employing alignment sequence CTF results in the fastest convergence. We employed the respective ASSM for all further experiments.

To evaluate the reconstruction capability of the knee ASSM, we conducted a leave-one-out study on 40 clinical MRI datasets that were used for model generation. To simulate the task of estimating healthy anatomy in the presence of pathologies, we manually labeled a region of interest around the joint gap (Fig. 2): In this region image features are not considered during model adaptation, i.e., the joint anatomy is purely extrapolated from the model. We compare the results to reconstructions obtained with single-object SSMs as in [2]. The results are presented in Tab. 1. We measured the accuracy by comparison to given manual expert segmentations in terms of Dice's coefficient (DICE), relative volume difference (RVD), average surface distance (AD), and average root mean square surface distance (RMS). 
Table 1. Average accuracy measures for ASSM and single-object SSM segmentation.

\begin{tabular}{lllll}
\hline & DICE & RVD & AD & RMS \\
\hline Femur (ASSM) & $0.94( \pm 0.02)$ & $0.05( \pm 0.03)$ & $1.12( \pm 0.28)$ & $1.52( \pm 0.44)$ \\
Tibia (ASSM) & $0.89( \pm 0.05)$ & $0.06( \pm 0.05)$ & $2.01( \pm 0.91)$ & $2.65( \pm 1.22)$ \\
Femur (Single) & $0.94( \pm 0.02)$ & $0.05( \pm 0.04)$ & $1.16( \pm 0.37)$ & $1.59( \pm 0.60)$ \\
Tibia (Single) & $0.86( \pm 0.10)$ & $0.12( \pm 0.12)$ & $2.61( \pm 2.08)$ & $3.52( \pm 2.77)$ \\
\hline
\end{tabular}

\section{Discussion}

The comparison between ASSM and two separate SSMs shows that both methods perform similar for the femur, whereas the reconstruction quality of the tibia was significantly improved by use of the ASSM (Fig. 2). The similar results for the femur might be attributed to its distinguished shape outside the extrapolated joint region: This seems to determine the extrapolated region sufficiently also for a femur SSM. In contrast, the shape of the tibia is less distinguished outside the extrapolated region. While the tibia SSM suffers from this ambiguity, the ASSM deals with it via the knowledge about the relative positioning of the tibia encoded in the joint model. Furthermore, part of the anterior tibia is ignored during adaptation by design of the profile analysis strategy [2], which adds to the above mentioned effect, but may also explain worse results for the tibia as compared to the femur for both methods. In summary, ASSMs are a promising tool for an accurate reconstruction of anatomical structures from poorly contrasted, incomplete or pathological medical image data.

Acknowledgement. This work was partially supported by the EU-FP7 Project MXL (ICT-2009.5.2), the DFG Research Center Matheon and the DFG Collaborative Research Center SFB760.

\section{References}

1. Cootes TF, Taylor CJ, Cooper DH, et al. Active shape models: their training and application. Comput Vis Image Underst. 1995;61(1):38-59.

2. Seim H, Kainmueller D, Lamecker H, et al. Model-based auto-segmentation of knee bones and cartilage in MRI data. In: Proc Med Image Anal; 2010. p. 215-23.

3. Klinder T, Wolz R, Lorenz C, et al. Spine segmentation using articulated shape models. Proc MICCAI. 2008; p. 227-34.

4. Heap T, Hogg D. Extending the point distribution model using polar coordinates. Image Vision Comput. 1996;14(8):589-99.

5. Kainmueller D, Lamecker H, Zachow S, et al. An articulated statistical shape model for accurate hip joint segmentation. In: Int Conf IEEE Eng Med Biol Soc; 2009. p. 6345-51.

6. Freeman MAR. How the knee moves. Curr Orthop. 2001;15(6):444-50.

7. Heimann T, Morrison BJ, Styner MA, et al. Segmentation of knee images: a grand challenge. In: Proc Med Image Anal; 2010. p. 207-14. 